\title{
The Leptonic CP Measurement and New Physics Alternatives
}

\author{
Shao-Feng Ge* \\ Tsung-Dao Lee Institute \& School of Physics and Astronomy, \\ Shanghai Jiao Tong University, Shanghai 200240, China \\ E-mail: gesfesjtu.edu.cn
}

\begin{abstract}
The accelerator-based measurement of the leptonic Dirac CP phase $\delta_{D}$ in neutrino mixing suffers from multiple intrinsic shortcomings. Even within the paradigm of three-neutrino oscillation with only the standard interactions predicted by the Standard Model (SM), accelerator neutrino experiments have several issues: 1) low efficiency and event rates, 2) $\delta_{D}$ vs $\pi-\delta_{D}$ degeneracy, and 3) large uncertainty for maximal $\mathrm{CP}, \delta_{D} \sim \pm \frac{\pi}{2}$ which is unfortunately or fortunately preferred by recent global fit. Going beyond the SM, new physics alternatives such as: 4) non-unitary mixing (NUM) and 5) non-standard interactions (NSI) can fake the CP violation effect and hence significantly reduce the sensitivity of the genuine Dirac CP phase. Especially, there is not just 5a) vector type NSI, but also 5b) scalar and 5c) dark NSI that can correct and fake the neutrino mass term in the effective Hamiltonian. The current and future accelerator-based experiments (such as T2K/T2K-II/T2HK/T2HKK, NOvA, and DUNE) can probably gather firm data, interpreting the Dirac CP phase out of it is significantly subject to theoretical assumptions. Unless extra experimental configuration is specifically designed to test these theoretical alternatives, no conclusive result can be reached. The TNT2K configuration, a combination of T2K/T2HK running purely on the neutrino mode and $\mu \mathrm{SK} / \mu \mathrm{HK}$ (muon decay at rest source plus the SK/HK detector) focusing on the antineutrino mode, can solve the aforementioned issues from item 1) to item 5a). For 5b) and $5 \mathrm{c}$ ), synergy of even more types of neutrino experiments.
\end{abstract}

The 21 st international workshop on neutrinos from accelerators (NuFact2019)

August 26 - August 31, 2019

Daegu, Korea

* Speaker. 


\section{Issues of CP Measurement in the Standard Three-Neutrino Oscillation}

The accelerator experiments use the neutrino-mode $v_{\mu} \rightarrow v_{e}$ and antineutrino-mode $\bar{v}_{\mu} \rightarrow \bar{v}_{e}$ oscillations for the $\mathrm{CP}$ measurement. Although the matter effect plays an important role, the basic features already appear in the vacuum oscillation probability expressions,

$$
\underset{\substack{P_{\nu_{\mu} \rightarrow v_{e}} \\ \bar{v}_{\mu} \rightarrow \bar{v}_{e}}}{ } \approx 4 s_{a}^{2} c_{r}^{2} s_{r}^{2} \sin ^{2} \phi_{31}-8 c_{a} s_{a} c_{r}^{2} s_{r} c_{s} s_{s} \sin \phi_{21} \sin \phi_{31}\left[\cos \delta_{D} \cos \phi_{31} \pm \sin \delta_{D} \sin \phi_{31}\right]
$$

where the \pm sign is for the neutrino and anti-neutrino modes, respectively. For convenience, we use shorthand notations $\left(\theta_{a}, \theta_{r}, \theta_{s}\right) \equiv\left(\theta_{23}, \theta_{13}, \theta_{12}\right)$ for the (atmospheric, reactor, solar) angles, $\left(c_{x}, s_{x}\right) \equiv\left(\cos \theta_{x}, \sin \theta_{x}\right)$, and $\phi_{i j} \equiv \Delta m_{i j}^{2} L / 4 E_{v}$ for the oscillation phases. To maximize the $v_{e} / \bar{v}_{e}$ event rates, accelerator-based experiments are usually configured to sit at the first oscillation peak, $\phi_{31} \approx \frac{\pi}{2}$ (T2K/T2K-II/T2HK, NOvA, DUNE), or the second one, $\phi_{31} \approx \frac{3 \pi}{2}$ (the second detector of T2HKK in Korea). A direct consequence is that $P_{v_{\mu} \rightarrow v_{e}}$ and $P_{\bar{v}_{\mu} \rightarrow \bar{v}_{e}}$ have only the $\sin \delta_{D}$ dependence while the $\cos \delta_{D}$ contribution is largely suppressed, introducing three intrinsic problems [1].

First, the CP term is extracted from the oscillation probability difference $P_{v_{\mu} \rightarrow v_{e}}-P_{\bar{v}_{\mu} \rightarrow \bar{v}_{e}} \propto$ $\sin \delta_{D}$. This approach is adopted not just because of the intuitive physics picture, but is necessary to avoid the large uncertainty from the first term in (1.1). The atmospheric angle $\theta_{a}$ can introduce via $s_{a}^{2}$ roughly $10 \%$ uncertainty which is even larger than the typical $5 \%$ effect of the CP term. Note that the relative size of the two terms, $2 c_{s} s_{S} \sin \phi_{21} / s_{r} \approx 2 / 5$, suppresses the $\sin \delta_{D}$ term. There is no choice but measuring both the neutrino and antineutrino modes if there is only acceleratorbased measurement. However, the cross section for the antineutrino mode is much smaller than the neutrino one. To gather comparable event rates for both modes, most of the run time should be assigned to the antineutrino mode. This essentially reduces the total event rate and enlarges the statistical uncertainties. In other words, the accelerator experiment for $\mathrm{CP}$ measurement is not efficient.

Second, the CP phase appears in the oscillation probabilities indirectly as $\sin \delta_{D}$. One can directly extract $\sin \delta_{D}$ from the oscillation data, but not $\delta_{D}$. The solution of $\delta_{D}$ from $\sin \delta_{D}$ is not unique, but has degeneracy between $\delta_{D}$ and $\pi-\delta_{D}$, where the $\chi^{2}$ curve would have two local minima. It is arguable that these two degenerate solutions converges for the maximal $\mathrm{CP}, \delta_{D}=$ $\pi-\delta_{D}= \pm \frac{\pi}{2}$ which is preferred by the recent global fit, and hence may not be a serious problem. Unfortunately, the maximal CP leads to a more serious problem in the CP uncertainty.

Third, the CP sensitivity is closely related to the variation of the oscillation probabilities with the CP phase $\delta_{D}$. The larger variation, the better CP sensitivity. With only $\sin \delta_{D}$ dependence, the variation has $\cos \delta_{D}$ dependence, $\partial P_{\mu e} / \partial \delta_{D} \propto \cos \delta_{D}$, which goes to zero for a maximal CP phase. The CP phase uncertainty is roughly proportional to the variation, $\Delta\left(\delta_{D}\right) \approx 1 / \cos \delta_{D}$. For the maximal CP phase, $\delta_{D} \approx \pm \frac{\pi}{2}$ and hence $\cos \delta_{D} \approx 0$, the CP uncertainty is intrinsically large. The current design of accelerator experiments, T2K/T2K-II/T2HK/T2HKK, NOvA, and DUNE, might be good enough for the discovery of a nonzero leptonic $\mathrm{CP}$, but definitely not for a precision measurement of its value especially for the maximal case.

The TNT2K configuration [2] can solve these three issues altogether. The first issue arises from splitting the run time while the other two by the $\sin \delta_{D}$-only dependence. These three issues can be solved with neutrinos produced in the muon decay at rest ( $\mu \mathrm{DAR})$. 
With a cyclotron delivering $800 \mathrm{MeV}$ low-energy proton beam to hit a thick target, the copiously produced $\pi^{-}$particles are absorbed by the target material while $\pi^{+}$first lose energy inside the target and then decay at rest, $\pi^{+} \rightarrow \mu^{+} v_{\mu}$. Similarly, $\mu^{+}$first loses its energy and decays at rest, $\mu^{+} \rightarrow e^{+} \bar{v}_{\mu} v_{e}$. Although three neutrinos $\left(v_{\mu}, \bar{v}_{\mu}, v_{e}\right)$ are produced, only $\bar{v}_{\mu}$ is useful for the $\mathrm{CP}$ measurement. This is because only the electron-flavor neutrinos can be uniquely probed via charged-current interactions while the muon-flavor neutrinos cannot produce muons in the final state due to lack of energy. Of $v_{e}$ and $\bar{v}_{e}$, only $\bar{v}_{e}$ can experience the inverse beta decay (IBD) which allows double coincidence for practical identification. These basically limits the oscillation channel to $\bar{v}_{\mu} \rightarrow \bar{v}_{e}$. Then, accelerator experiments can focus on the neutrino mode while the $\mu$ DAR component can focus on the antineutrino mode. From T2K/T2HK to TNT2K/TNT2HK, the neutrino event rate increases by a factor of 3 while the antineutrino one by a factor of 7 , significantly improving the efficiency.

Second, the $\mu$ DAR $\bar{v}_{\mu}$ spectrum is much wider than the accelerator neutrino spectrum and extends up to $53 \mathrm{MeV}$. With such a wide spectrum, there is no way to hide the $\cos \delta_{D}$ term. Since the items 2) and 3) in the abstract arise due to the absence of the $\cos \delta_{D}$ term, introducing a $\mu$ DAR component to accelerator experiments can basically remove the $\delta_{D}$ vs $\pi-\delta_{D}$ degeneracy and significantly reduce the $\mathrm{CP}$ uncertainty around the maximal $\mathrm{CP}$ value.

\section{Issues of CP Measurement beyond the Standard Case}

Experiment should be treated as a black box before opening it to reduce subjective influences. But this is not enough to guarantee that the result is really free of prejudice. The interpretation of data is always subject to the theoretical assumption that it is based on. The measurement of the leptonic Dirac CP phase provides a very good example of this. We shall keep reminding ourselves of theoretical alternatives when talking about the $\mathrm{CP}$ sensitivity from the accelerator-based neutrino oscillation experiments.

First, the non-unitary mixing extends the degrees of freedom in the neutrino mixing matrix,

$$
N=N^{N P} U=\left(\begin{array}{ccc}
\alpha_{11} & 0 & 0 \\
\alpha_{21} & \alpha_{22} & 0 \\
\alpha_{31} & \alpha_{32} & \alpha_{33}
\end{array}\right) U,
$$

where $U$ is the $3 \times 3$ unitary mixing matrix and $N^{N P}$ parametrizes the NUM parameters. For $\mu \rightarrow e$ transition, the phase in $\alpha_{21} \equiv\left|\alpha_{21}\right| e^{-i \phi}$ can fake CP effect [3],

$$
\begin{aligned}
P_{\mu e}^{\mathrm{NUM}} & =\alpha_{11}^{2}\left\{\alpha_{22}^{2}\left[c_{a}^{2}\left|S_{12}^{\prime}\right|^{2}+s_{a}^{2}\left|S_{13}^{\prime}\right|^{2}+2 c_{a} s_{a}\left(\cos \delta_{D} \mathbb{R}-\sin \delta_{D} \mathbb{I}\right)\left(S_{12}^{\prime} S_{13}^{\prime *}\right)\right]+\left|\alpha_{21}\right|^{2} P_{e e}\right. \\
& \left.+2 \alpha_{22}\left|\alpha_{21}\right|\left[c_{a}\left(c_{\phi} \mathbb{R}-s_{\phi} \mathbb{I}\right)\left(S_{11}^{\prime} S_{12}^{*}\right)+s_{a}\left(c_{\phi+\delta_{D}} \mathbb{R}-s_{\phi+\delta_{D}} \mathbb{I}\right)\left(S_{11}^{\prime} S_{13}^{\prime *}\right)\right]\right\} .
\end{aligned}
$$

In addition to the genuine $\mathrm{CP}$ terms $\left(\cos \delta_{D}, \sin \delta_{D}\right)$, four extra terms $\left(c_{\phi}, s_{\phi}\right)$ and $\left(c_{\phi+\delta_{D}}, s_{\phi+\delta_{D}}\right)$ appear to significantly reduce the CP sensitivity at T2(H)K. As shown in the left plot of Fig. 1 , the $\chi^{2}$ curves become flat once introducing the NUM, which means that the CP sensitivity can completely disappear. Even firm data is obtained, no knowledge about the CP phase can be extracted if the analysis framework slightly extends. Fortunately, adding a 20 ton near detector around the $\mu$ DAR source for measuring the zero-distance effect, $\left.P_{\mu e}^{\mathrm{NUM}}\right|_{L \rightarrow 0} \rightarrow\left|\alpha_{21}\right|^{2}$, to suppress the size $\left|\alpha_{21}\right|$ of the extra CP term to permille level can fully restore the CP sensitivity, shown as the right plot of Fig.1. 

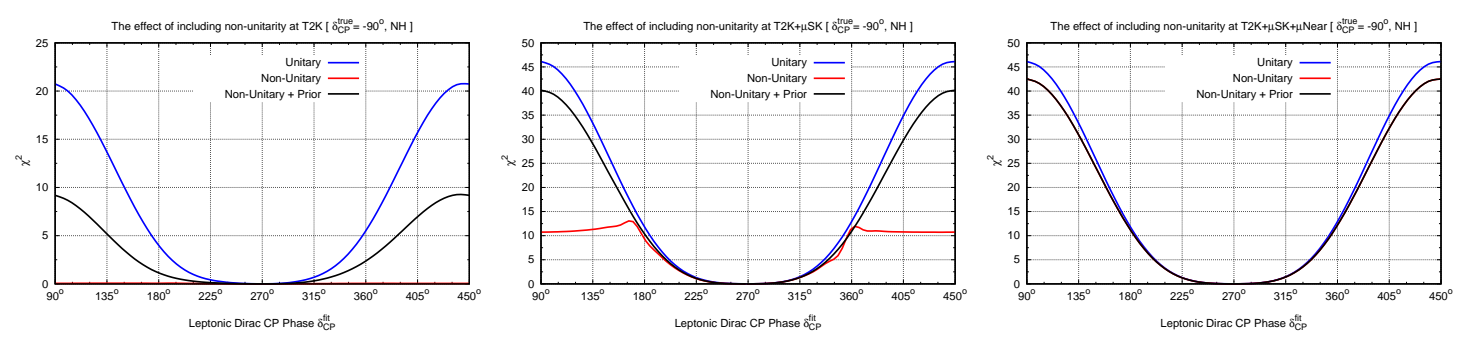

Figure 1: The $\mathrm{CP}$ sensitivity with the NUM at (Left) $\mathrm{T} 2 \mathrm{~K}$, (Middle) $\mathrm{T} 2 \mathrm{~K}+\mu \mathrm{SK}$, and (Right) $\mathrm{T} 2 \mathrm{~K}+\mu \mathrm{SK}+\mu \mathrm{Near}$.

Second, the vector NSI can also introduce extra parameters $\varepsilon_{\alpha \beta}$,

$$
\mathscr{H} \equiv \frac{1}{2 E_{v}} U\left(\begin{array}{lll}
0 & & \\
& \Delta m_{s}^{2} & \\
& & \\
& & \Delta m_{a}^{2}
\end{array}\right) U^{\dagger}+V_{c c}\left(\begin{array}{ccc}
1+\varepsilon_{e e} & \varepsilon_{e \mu} & \varepsilon_{e \tau} \\
\varepsilon_{e \mu}^{*} & \varepsilon_{\mu \mu} & \varepsilon_{\mu \tau} \\
\varepsilon_{e \tau}^{*} & \varepsilon_{\mu \tau}^{*} & \varepsilon_{\tau \tau}
\end{array}\right) .
$$

Even the real diagonal elements $\varepsilon_{\alpha \alpha}$ can already fake the CP effect. Similar to the NUM case, the $\chi^{2}$ curve can reduce a lot [4], as demonstrated in Fig. 2. Fortunately, the effect of vector
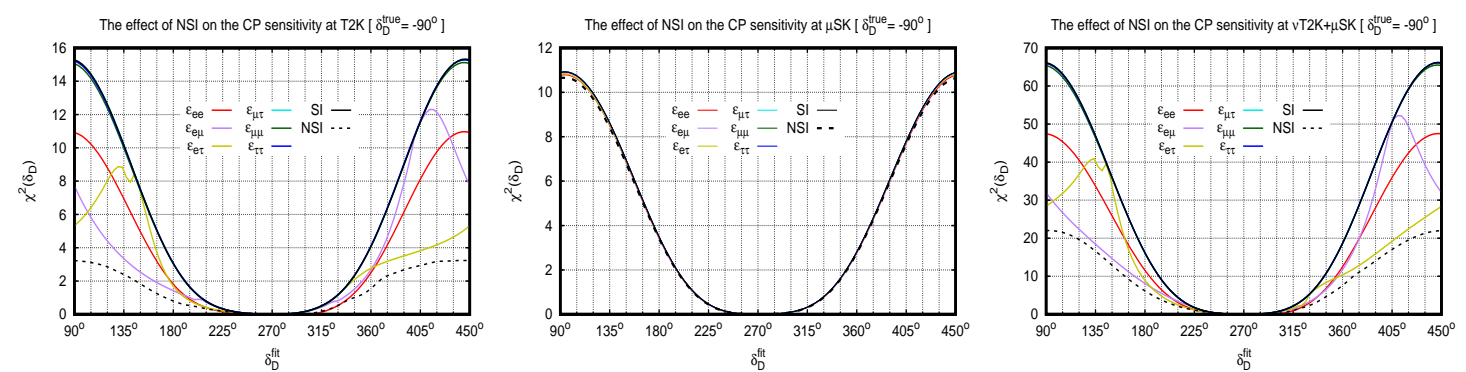

Figure 2: The CP sensitivity with the vector NSI at (Left) $\mathrm{T} 2 \mathrm{~K}$, (Middle) $\mu \mathrm{SK}$, (Right) $v \mathrm{~T} 2 \mathrm{~K}+\mu \mathrm{SK}$.

NSI on neutrino oscillation is proportional to the neutrino energy $E_{v}$. Since the $\mu$ DAR neutrino spectrum has much lower energy, the $\mu \mathrm{SK} / \mu \mathrm{HK}$ is sensitive to only the genuine $\mathrm{CP}$ phase while the $\mathrm{T} 2 \mathrm{~K} / \mathrm{T} 2 \mathrm{HK}$ part is sensitive to both the genuine one and the vector NSI. This combination provides two independent measurements to disentangle the two effects, namely measuring both of them, and hence can help to restore the $\mathrm{CP}$ sensitivity.

Finally, the scalar NSI [5] and dark NSI [6] can fake the neutrino mass term,

$$
\mathscr{H}=\frac{\left(M+\delta M_{S}\right)\left(M+\delta M_{S}\right)^{\dagger}+\delta M_{D}^{2}}{2 E_{V}} \pm V_{\mathrm{SI}} \pm V_{\mathrm{NSI}}
$$

where $\delta M_{S}$ is the scalar NSI correction while $\delta M_{D}^{2}$ is the dark NSI contribution. Together with the conventional vector NSI contribution, $V_{\mathrm{NSI}}$, this basically exhausts the possible corrections to the effective Hamiltonian for neutrino oscillation.

While the vector NSI appears as correction to the standard matter potential $\left(V_{\mathrm{SI}}\right)$, the scalar and dark types modify the neutrino mass term. In principle, the neutrino mass term needs not to be a genuine one but just some environmental effect, especially for the dark NSI. It is completely fine to turn off the genuine neutrino mass term $M$ and just let $\delta M_{D}^{2}$ to drive the neutrino oscillation. 
Since the dark matter density is pretty homogeneous inside our solar system, the effective neutrino mass $\delta M_{D}^{2}$ can fit the neutrino oscillation data quite well since all neutrino oscillation experiments up to now are done locally within the solar system. Neutrino oscillation does mean that there is some new physics beyond the SM, but it is not necessarily a genuine neutrino mass term.
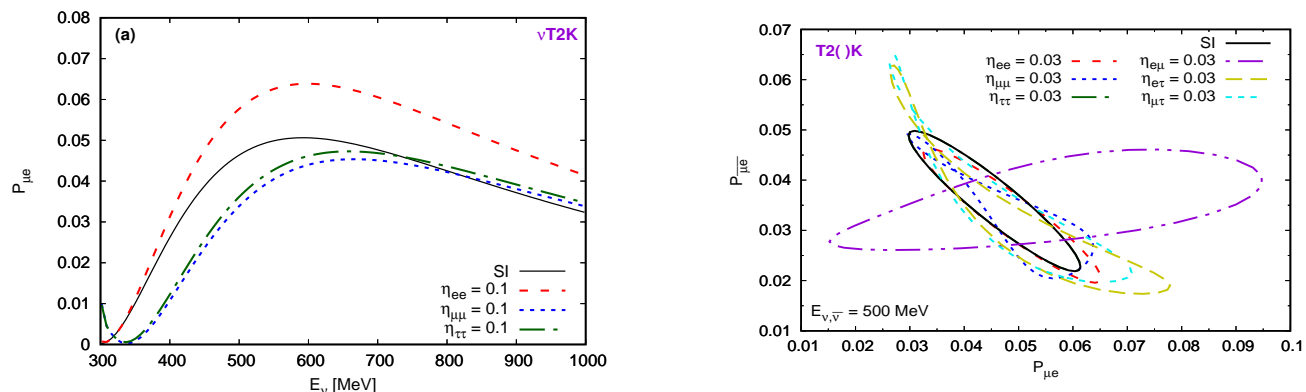

Figure 3: The effect of the scalar (left) and dark (right) NSI on $P_{\mu e}$.

Since the scalar or dark NSI can fake the neutrino mass matrix, they can affect everything including mixing angles and mass squared differences. For the CP phase, Fig. 3 shows how the oscillation probabilities can be significantly modified by the scalar and dark NSI's. It is very important to find experimental solutions to guarantee that we can measure the genuine $\mathrm{CP}$ phase.

We can imagine that once one of T2K/T2HK, NOvA, and DUNE reaches $5 \sigma$ sensitivity for the CP discovery, the community would seriously think about the possibility of giving them a Nobel Prize. But if theoretical alternatives have not been experimentally excluded then, it would become a serious problem for the neutrino community to convince ourselves and other relevant parties that we have really measured the genuine leptonic Dirac CP phase. We need not just firm data but also firm interpretation.

\section{Acknowledgments}

This study is supported by JSPS KAKENHI Grant Number JP18K1353.

\section{References}

[1] S. F. Ge, "Measuring the Leptonic Dirac CP Phase with TNT2K," [arXiv:1704.08518 [hep-ph]].

[2] J. Evslin, S. F. Ge and K. Hagiwara, "The leptonic CP phase from T2(H)K and $\mu^{+}$decay at rest," JHEP 1602, 137 (2016) [arXiv:1506.05023 [hep-ph]].

[3] S. F. Ge, P. Pasquini, M. Tortola and J. W. F. Valle, "Measuring the leptonic CP phase in neutrino oscillations with nonunitary mixing," Phys. Rev. D 95, no. 3, 033005 (2017) [arXiv:1605.01670 [hep-ph]].

[4] S. F. Ge and A. Y. Smirnov, "Non-standard interactions and the CP phase measurements in neutrino oscillations at low energies," JHEP 1610, 138 (2016) [arXiv:1607.08513 [hep-ph]].

[5] S. F. Ge and S. J. Parke, "Scalar Nonstandard Interactions in Neutrino Oscillation," Phys. Rev. Lett. 122, no. 21, 211801 (2019) [arXiv:1812.08376 [hep-ph]].

[6] S. F. Ge and H. Murayama, "Apparent CPT Violation in Neutrino Oscillation from Dark Non-Standard Interactions,” [arXiv:1904.02518 [hep-ph]]. 